\title{
Increased expression of CD20 and CD45 and diminished expression of CDI9 are features of follicular lymphoma
}

This article was published in the following Dove Press journal:

Pathology and Laboratory Medicine International

30 May 2013

Number of times this article has been viewed

\author{
Qingyan Liu' \\ Linda S Weaver' \\ David Liewehr ${ }^{2}$ \\ David Venzon ${ }^{2}$ \\ Maryalice Stetler-Stevenson' \\ Constance M Yuan' \\ 'Flow Cytometry Unit, Laboratory \\ of Pathology, ${ }^{2}$ Biostatistics and Data \\ Management Section, National Cancer \\ Institute, National Institutes of Health, \\ Bethesda, MD, USA
}

Correspondence: Constance M Yuan Flow Cytometry Unit, Laboratory of Pathology, 10 Center Drive, MSC - I500, Bethesda, MD 20892-1500, USA

Tel +l 30I 4964709

Fax +I 30I 4020043

Email yuanc@mail.nih.gov

\begin{abstract}
Follicular lymphoma (FL) is a relatively common, well-characterized lymphoma with recognizable morphologic and immunophenotypic features. Nevertheless, close examination of its immunophenotypic profile by mean fluorescent intensity (MFI) and potential relationship between antigens, as well as histologic grade, has not been extensively assessed by flow cytometry immunophenotyping (FC). We examined the immunophenotypic profile, including heavy and light chain analysis, of 41 nodal FL cases with FC analysis and tissue diagnosis. Additionally, MFI of CD45, CD19, CD20, CD22, and CD10 were examined. The relationship between the antigen expression on FL cells and normal B cells in the same sample, and any association with each other or with histologic grade, were analyzed statistically. We observed brighter CD45 and CD20 expression in FL than normal B cells $(P<0.0001)$; dimmer CD19 in FL than normal B cells $(P=0.03)$; brighter CD20 in grade 2 than grade 1 histology $(P=0.0023)$. No correlation was observed between CD10 MFI and other antigens. Increased expression of CD20, CD45, and dim expression of CD19 are features of nodal FL. FC assessment of MFI detects subtle changes in antigen expression not entirely apparent by visual examination of dot plots or immunohistochemistry. MFI is a means of uncovering subtle but unique immunophenotypic features in both well-known, and less well-defined neoplastic hematolymphoid entities.
\end{abstract}

Keywords: lymphoma, lymphoproliferative disorder, flow cytometry, mean fluorescent intensity, immunophenotyping, immunoglobulin heavy chain

\section{Introduction}

Follicular lymphoma (FL) is a common lymphoma, accounting for approximately $20 \%$ of all lymphomas, with the highest incidence in the United States and western Europe. ${ }^{1}$ Diagnosis of FL relies on characteristic histologic features and immunohistochemical profile, supported by other ancillary studies, such as flow cytometry analysis (FC), cytogenetic, and molecular studies. However, diagnosis can be challenging if atypical morphology, minimal involvement or unusual immunophenotype is encountered. Furthermore, florid follicular hyperplasia may mimic many features of FL. In addition, diagnosis is increasingly reliant on fine needle aspiration, a smaller sample compared to the traditional lymph node (LN) biopsy. Although identification of $\mathrm{t}(14 ; 18)$ translocation or B-cell clonality by polymerase chain reaction (PCR) can be helpful, not all cases possess the translocation or exhibit identifiable B-cell clonality. ${ }^{1-3}$ The $t(14 ; 18)$ translocation may be detected in healthy individuals without evidence of lymphoma. ${ }^{4}$ Furthermore, B-cell clonality identified by PCR may be observed in reactive conditions. ${ }^{5}$ In these scenarios, FC is useful in diagnosis and subclassification of non-Hodgkin's lymphoma by identifying clonal B-cells with 
aberrant antigen expression. Expression of CD10, Bcl-2, along with mature B-cell markers in a monoclonal B-cell population is a well-recognized feature of FL. Abnormally increased or decreased antigen intensity is a hallmark of lymphoma cells ${ }^{6,7}$ and changes in CD19 and CD38 expression have been described in FL. ${ }^{8,9}$ Nevertheless, examination of the antigen profile and intensity of antigen expression, potential relationship between antigens, and possible correlations to histologic grade, has not been thoroughly assessed by mean fluorescent intensity (MFI) by flow cytometry. Our study seeks to examine additional useful immunophenotypic markers and their potential relationship with each other, and with histologic grade, to be utilized in improving the characterization and diagnosis of FL.

\section{Materials and methods Case selection}

Forty-one nodal FL cases were received in the Flow Cytometry Laboratory, Laboratory of Pathology, National Cancer Institute (NCI), National Institutes of Health (NIH) (Bethesda, MD, USA) between 2006 and 2009. These included 2/41 LN fine needle aspirates and 39/41 LN cell suspensions. The median age was 41 years, with a male to female ratio of 1:1 (20 males, 20 females, one sex unknown). Thirty-eight of 41 cases had FC analysis and corresponding in-house tissue diagnosis performed at the Flow Cytometry Unit and Hematopathology section of the Laboratory of Pathology, NCI/NIH, with the exception of three cases without corresponding in-house tissue diagnosis.

Cases with corresponding tissue diagnosis were graded according to World Health Organization Classification of Tumors of Hematopoietic and Lymphoid Tissues. ${ }^{1}$ Of three of the 41 remaining cases, one case had only a bone marrow biopsy reviewed at our institute. The diagnosis of FL was made on a $\mathrm{LN}$ that was reviewed at an outside institution. The bone marrow was diagnosed as consistent with involvement by FL. In the other two cases, flow cytometry was performed as a part of routine follow-up. Corresponding in-house tissue diagnosis was not available in these two cases; however, the diagnosis of FL was rendered by prior LN biopsies performed several years before, and confirmed upon in-house review. Both cases were diagnosed as FL grade 1 of 3 .

\section{Immunophenotyping by flow cytometry}

Four-color FC was performed on all 41 specimens as described previously. ${ }^{10}$ Samples included fine needle aspirations and LN cell suspensions. The LN cell suspension was either prepared in-house (10/41 cases) or off-site (31/41 cases). In the latter situation, the samples were stored frozen, and shipped to our laboratory on dry ice. Specimens were washed with phosphate-buffered saline to remove cytophilic antibodies before determining cell numbers. Cellularity was manually determined using a hemocytometer and viability was determined by trypan blue uptake. Erythrocytes were lysed by incubating with the lysing solution $\left(150 \mathrm{mM} \mathrm{NH}_{4} \mathrm{Cl}, 10 \mathrm{mM}\right.$ $\mathrm{KHCO}_{3}$, and $0.1 \mathrm{mM}$ ethylenediaminetetraacetic acid) for 10 minutes at room temperature at a ratio of 1:9 (volume of sample:volume of lysing solution).

Specimens were stained for 30 minutes at room temperature with a cocktail of four antibodies (antibody concentration according to manufacturer's recommendations). The following fluorochromes were utilized: fluorescein isothiocyanate (FITC), phycoerythrin (PE), peridinin-chlorophyll-protein complex (PerCP), and allophycocyanin (APC). The following panel was used (as listed in the fluorochrome order, FITC/PE/ PerCP/APC): CD14/CD56+CD16/CD19/CD3, CD4/CD8/ CD45/CD3, CD5/CD38/CD19/CD34, CD20/CD22/CD45/ CD10, CD103/CD25/CD20/CD11c, FMC7/CD79b/CD19/ CD23, א-monoclonal/lambda-monoclonal/CD19/CD5, $\kappa$-polyclonal/CD22/CD20/CD10, $\lambda$-polyclonal/CD22/CD20/ CD10, immunoglobulin A (IgA)/mouse IgG2a/CD19/CD10, IgD/mouse IgG2a/CD19/CD10, IgG/mouse IgG2a/CD19/ CD10, IgM/mouse IgG2a/CD19/CD10. Antibody sources are as follows: CD3, FMC7, CD79b (Beckman-Coulter, Inc, Miami, FL, USA); CD20, CD103, antibodies to the heavy chains, mouse IgG2a, $\kappa$-polyclonal, $\lambda$-polyclonal (Dako North America, Inc, Carpenteria, CA, USA); all others (Becton, Dickinson, and Company, San Jose, CA, USA). After incubation, the cells were pelleted by centrifugation ( $500 \times g$ for 5 minutes at room temperature), the media was aspirated, and the cells were washed twice in a phosphatebuffered saline solution containing $0.1 \%$ sodium azide $\left(\mathrm{NaN}_{3}\right)$, and fixed in $1.0 \%$ paraformaldehyde.

Data was acquired using a FACSCalibur flow cytometer equipped with CellQuest software (Becton Dickinson). Sensitivity of fluorescent detectors was monitored using standard beads according to the manufacturer's recommendations. For acquisition, a minimum of 5000 events falling within the lymphoid region by forward scatter (FSC) and side scatter (SSC) were collected per tube. Data were analyzed using FCS Express v3 software (De Novo Software, Los Angeles, CA, USA), by gating on cells consistent with lymphoid cells by FSC and SSC, and used also to exclude debris. Antigen back-gating for T-cells (CD3) and B-cells (CD19) ensured that the gating appropriately captured the cells of interest for evaluation. Normal lymphoid cells 
within specimens served as internal controls, as well as for antibody-binding intensity. Bright expression was defined as higher than observed in normal lymphocytes while dim expression was defined as lower than that observed in normal lymphocytes, in concordance with the 1997 US-Canadian consensus guidelines. ${ }^{11}$

\section{Assessment of mean fluorescence intensity of CDI0, CD 19, CD20, CD22, and CD45 between FL and non-FL B-cells}

The MFI of various surface markers was analyzed on 39 of 41 cases (in two cases, flow list mode data was not accessible for analysis). The population of FL cells was defined by B-cell markers, CD10 positivity, and light chain restriction, and used to differentiate them from germinal center B-cells, if present. In one case in which the anti-light chain antibodies utilized for analysis failed to identify definitive monoclonal light chain expression clearly, the population of FL cells was identified by IgG heavy chain expression. For FL cases that did not express CD10, the FL cells were defined by CD19/ CD20 positivity and light chain restriction. The cellularity of the cases was composed of $88 \%$ FL cells on average, consistent with extensive involvement of the LN by FL. All of the cases contained residual, nonneoplastic (non-FL) B-cells, which represented $13 \%$ of B-cells on average among our series of cases. Nonneoplastic cells (non-FL B-cells) present within each sample were utilized to compare the MFI of various antigens between FL and non-FL B-cells. The antigen/antibodyfluorochrome combination used for MFI was driven by the antibody combinations available. The best combination that allowed distinguishing FL from non-FL B-cells was used for the antigen of interest to determine MFI. To analyze the MFI of CD19-PerCP, the tube containing IgD, IgM, IgG, and IgA/ mouse IgG2a/CD19/CD10 was used. To analyze the MFI of CD20-PerCP, CD22-PE, and CD45-PerCP, the tube containing CD20/CD22/CD45/CD10 was used. MFI of each antigen was determined for both FL and non-FL B-cells. The MFI of these markers was analyzed for any relationship or association between FL and non-FL B-cells (see Statistical analysis).

\section{MFI of various antigens expressed on FL cells: potential associations with each other and with histologic grade}

The MFI of CD19, CD20, CD22, CD45, and CD10 in FL cells was examined for any potential associations between intensity of expression with each other, as well as with the reported histologic grade. The MFI of the antigens on FL cells was obtained from the tubes described above. For CD10, either the $\kappa-\mathrm{CD} 22 / \mathrm{CD} 20 / \mathrm{CD} 10$ tube, or the $\lambda-\mathrm{CD} 22 / \mathrm{CD} 20 /$ $\mathrm{CD} 10$ tube was used, as appropriate for each individual case. For FL cases that expressed dim or negative CD10, the FL cells were defined by CD19/CD20 positivity and light chain restriction.

\section{Statistical analysis}

We investigated any potential differences in the MFI of antigens between FL cells and nonneoplastic (non-FL) B cells. Four antigens (CD19, CD20, CD22 and CD45) in two cell types (FL cells and normal B cells) were analyzed. Preliminary analyses indicated that a $\log 10$ (MFI) transformation would make the data more consistent with the assumptions of analysis of variance (ANOVA). Thus, repeated measures ANOVA were performed on the transformed data.

To investigate the potential relationship between the MFI of CD10 and MFI of other antigens (CD45, CD19, CD20 and CD22) on FL cells, Spearman's rank correlation coefficient was applied on $\log$ transformed data. Finally, the Wilcoxon rank sum test was used to compare the distributions of MFI of CD10, CD45, CD19, CD20 and CD22 to the histology grade (grade 1 versus grade 2). Too few grade 3 cases were available in our series for meaningful comparison.

\section{Results \\ Immunophenotype}

Among our 41 specimens, the percent of lymphocytes that were comprised of T-cells averaged $33.3 \%( \pm 18.6 \%, 1$ standard deviation $[\mathrm{SD}]$ ), and ranged from $12 \%-88 \%$ of lymphocytes overall. The percent of lymphocytes that were comprised of B-cells averaged $66.6 \%( \pm 18.9 \%, 1 \mathrm{SD})$, and also ranged from $12 \%-88 \%$ of lymphocytes. The percent of lymphocytes that were comprised of NK-cells was consistently less than $1 \%$ among all of the specimens. Of the B-cell compartment, $87.6 \%( \pm 13.6 \%, 1 \mathrm{SD})$ of B-cells in the specimens studied were lymphoma cells, with percent of lymphoma ranging from $37 \%$ to $98 \%$. Regarding the B-cell compartment for our series of cases, the percent of normal bystander B-cells ranged from $2 \%-63 \%$, and averaged $13 \%$ of the total B-cells.

Immunophenotypically, $100 \%(41 / 41)$ of the cases expressed CD19, CD20, CD22, CD45, and were negative for CD5, CD13, CD103, and CD11c (Figure 1, representative case). 98\% (40/41) cases were CD25 negative, and one case showed dim CD25 expression. All 37 cases assessed for CD79b expression were positive for CD79b, and the remaining 4/41 cases did not have CD79b assessed. 98\% (39/40) of the cases 

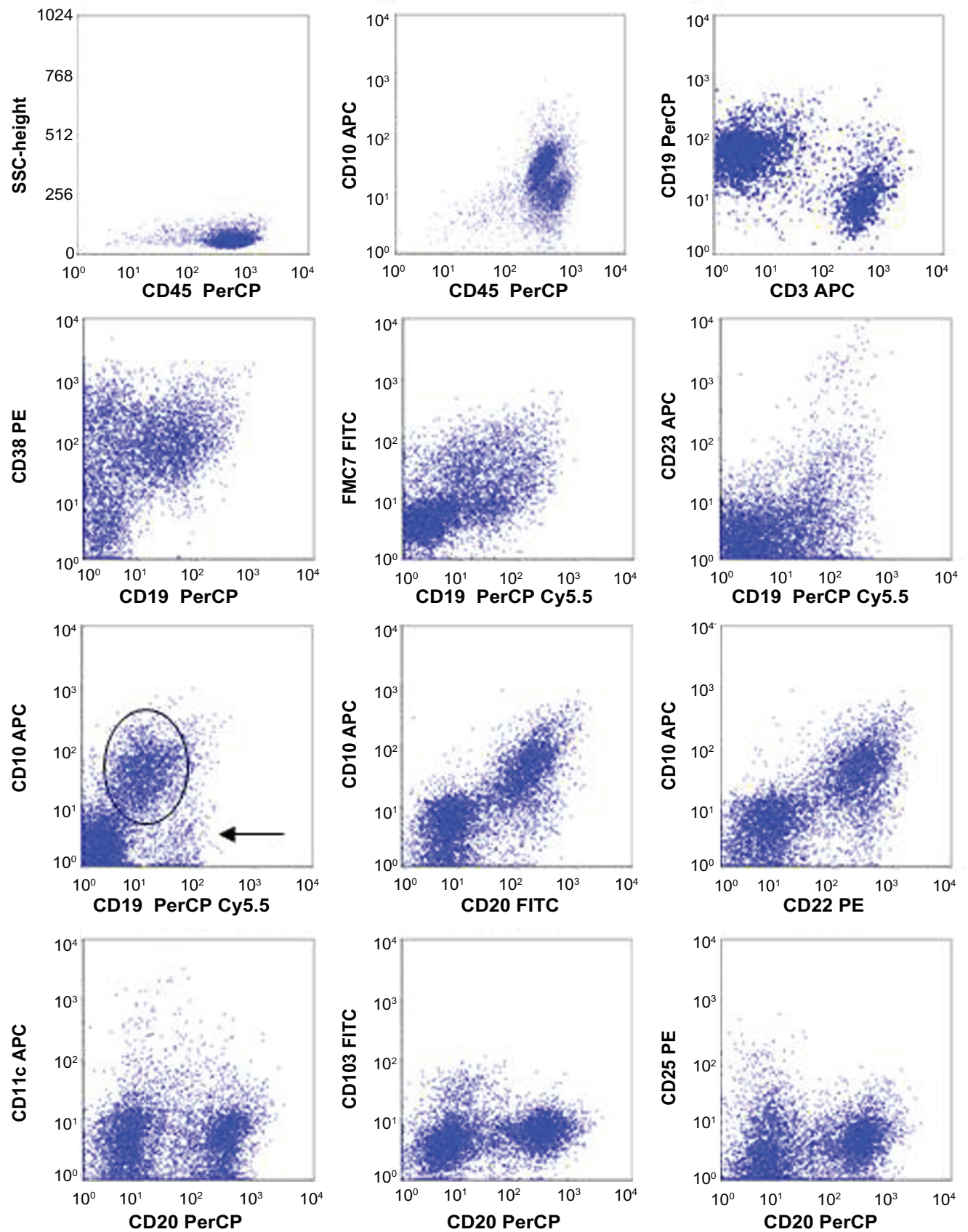

Figure I Flow cytometry analysis of a representative case of nodal follicular lymphoma (FL).

Notes: By four-color FC, The FL cells express dim CDI9, CD20, CD22, CDI0, CD38, FMC7, and CD45. The FL cells are negative for CD23, CDI03, CDI I c, and CD25. The $\operatorname{dim}$ CDI 9 expression is observed on the CDIO(+) FL cells (indicated by the oval), and is easily recognized when compared to CDI0(-) B cells (arrow) within the sample. Abbreviations: FITC, fluorescein isothiocyanate; PE, phycoerythrin; PerCP, peridinin-chlorophyll-protein complex; APC, allophycocyanin.

expressed CD38 on B cells. Regarding CD23 and FMC7 antigens, 54\% (22/41) of the cases expressed CD23, and 90\% (37/41) expressed FMC7. The expression of CD23 was often heterogeneous, showing a spectrum of intensity ranging from moderate to dim. Regarding CD10 expression on FL cells, 98\% (40/41) of the cases were CD10(+), 10\% (4/40) of which were partially CD10(+), and 2.5\% (1/41) were CD10(-).

Regarding immunoglobulin expression, all but one of the cases showed light chain restriction with $56 \%$ expressing kappa light chain (23/41) and 41\% expressing lambda light chain (17/41); the single exception was a case lacking surface light chain expression altogether. 40/41 cases had heavy chain expression examined (Table 1). We observed that 52\% expressed IgM (21/41), 38\% expressed IgG (15/41), and 3\% expressed IgA (1/41); none of the cases expressed IgD only. Interestingly, we observed occasional cases where more than one immunoglobulin heavy chain was expressed on the FL cells. We identified one case (3\%) with kappa light chain 
restriction where the FL cells exhibited co-expression of both IgM and dim IgD heavy chain (Figure 2A; histologic grade 1 of 3 ). Additionally, we identified 2 cases (5\%) (histologic grade 1/3 and histologic grade 2/3) with lambda light chain restriction where a subpopulation of the FL cells expressed IgM heavy chain and a subpopulation expressed IgG heavy chain (Figure 2B, representative case).

\section{Histologic grade}

38/41 FL cases consisted of in-house material that was histologically evaluated and graded; in the remaining $2 / 41$ cases, in-house review of outside LN biopsy confirmed the diagnosis of FL, grade 1 of 3 for both of these cases.

We found that $36 / 38$ cases $(95 \%)$ were grade $1-2$ of 3 (including 16 grade 1 cases, 18 grade 2 cases, 2 grade $1-2$ cases), and $2(5 \%)$ were grade $3 a$ of 3.
Regarding CD10 expression and its association with histologic grade, $100 \%(36 / 36)$ of grade $1-2$ cases were $\mathrm{CD} 10(+), 11 \%(4 / 36)$ of which were partially CD10(+). Among the two grade $3 \mathrm{a}$ cases, one was $\mathrm{CD} 10(+)$, and the other was CD10(-).

\section{Assessment of MFI of CD19, CD20, CD22, CDIO and CD45 on FL and non-FL cells}

In 39/41 cases, the MFI of CD19, CD20, CD22 and CD45 on FL and normal B-cells was determined, and any association between the MFI of FL and normal B-cells was examined (Figures 3 and 4). CD20 and CD45 expression on FL cells was significantly greater than that of normal B-cells within the same sample $(P<0.0001)$. Stronger $\mathrm{CD} 20$ and CD45 expression on FL cells in comparison to

A
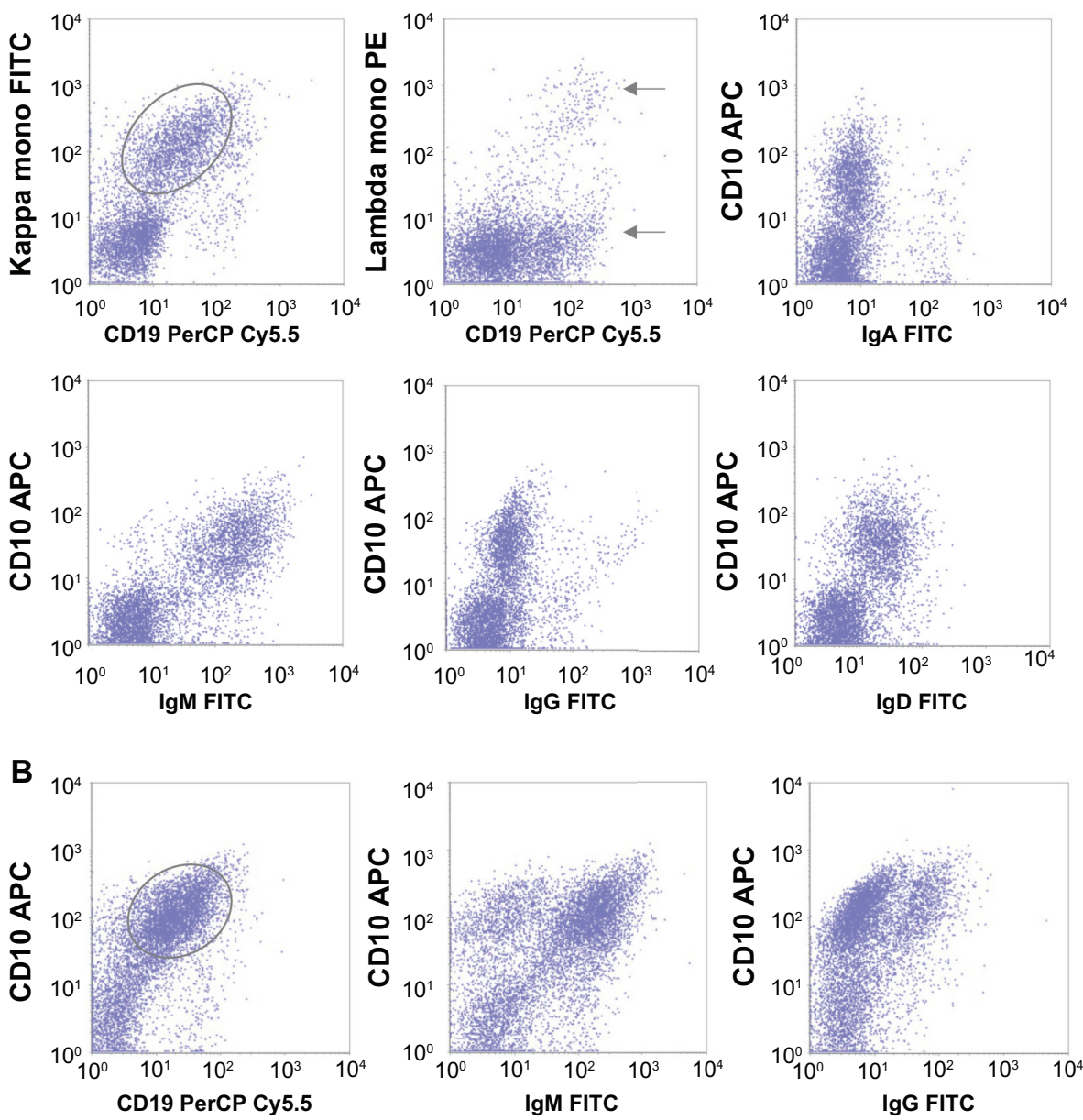

Figure 2 Flow cytometry analysis of two follicular lymphoma (FL) cases with coexpression of more than one immunoglobulin heavy chain. In one $\mathrm{FL}$ case (A), the $\mathrm{FL}$ cells (indicated by oval) are CDI9 $\operatorname{dim}(+), \mathrm{CDIO}(+), \kappa$ light chain-restricted, and co-express immunoglobulin $\mathrm{M}$ (IgM) and IgD heavy chain (lgA and lgG are negative). Few residual polyclonal B-cells are present, with normal intensity of CDI9 expression (arrows). In another FL case (B), the FL cells (indicated by oval) are CDI9 dim(+), CDI0(+), and show expression of both IgM and IgG heavy chain, with $63 \%$ expressing IgM and $24 \%$ expressing IgG.

Abbreviations: FITC, fluorescein isothiocyanate; PE, phycoerythrin; PerCP, peridinin-chlorophyll-protein complex; APC, allophycocyanin. 


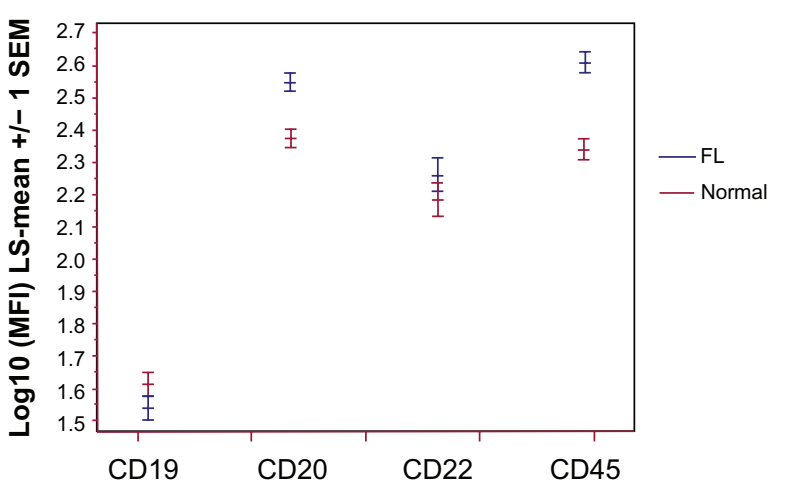

Figure 3 Mean fluorescent intensity (MFI) of CDI9, CD20, CD22, and CD45 on follicular lymphoma cells (FL) and nonneoplastic B-cells (normal).

Notes: MFI of various surface markers was determined and analyzed statistically as described (see Material and Methods). The least squares (LS) means are shown (ie, predicted values from the best fitting model), \pm I standard error of the mean (SEM), for each cell population type (blue, FL cells; red, normal cells), for each antigen (eg, CDI9, CD20, CD22, CD45). Note that the means are on the $\log 10$ scale. The intensity of expression of CD20, CD22, and CD45 in FL cells is increased when compared to nonneoplastic B-cells within the sample. The intensity of expression of CDI9 in FL cells is decreased when compared to nonneoplastic B-cells within the sample.

normal B-cell was observed in FL cases, regardless of the intensity of CD10 expression. On the other hand, CD19 expression on FL cells was weaker than that of normal B-cells $(P=0.033)$. CD22 expression on FL cells exhibited greater variability in MFI. Although CD22 initially appeared to exhibit slightly higher intensity of expression in FL cells compared to normal B-cells (Figures 3 and 4), CD22 expression varied, and ultimately was not statistically significant. $(P=0.081)$. A strong correlation among MFI of CD19, CD20 and CD22 was also observed on both
FL and normal B-cells (Spearman rank correlation $\geq 0.5$, $P<0.003$ for each). This did not appear to involve the histologic grade (data not shown).

\section{Association of MFI of CDI9, CD20, CD22, CD45 and CDI0 with histologic grade}

The association of MFI of CD19, CD20, CD22, CD45 and CD10 with histologic grade 1 versus grade 2 was determined using Wilcoxon rank sum test. No significant association was detected between MFI of CD19, CD22, CD45, CD10 and the histologic grade $(P=0.68,0.17$, $0.92,0.36$, respectively). A significant difference in the MFI of CD20 expression and histologic grade 1 versus grade 2 was detected, ie, the CD20 expression was stronger in grade 2 cases than in grade 1 cases $(P=0.0023)$. A similar relationship was also observed in normal $\mathrm{B}$ cells $(P=0.019)$.

\section{Association of MFI of CDI0 with CDI9, CD20, CD22 and CD45 in FL cells}

Using Spearman's rank correlation coefficient (r), we tested for an association between CD10 MFI and the following MFI variables (in FL cells): CD45, CD19, CD20 and CD22. No significant association could be detected between the MFI of each antigen. All of the correlation coefficients were such that $|\mathrm{r}|<0.42$; thus, all of the correlations were at best weak, and the $P$ values, corrected for multiple tests, were $>0.05$.

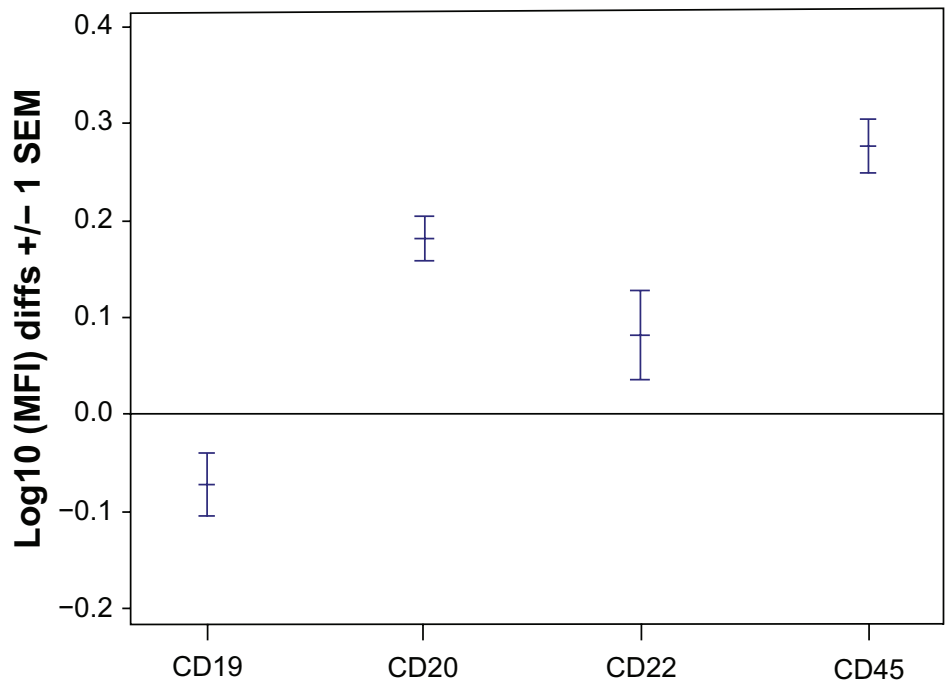

Figure 4 Ratio of mean fluorescent intensity (MFI) of CDI9, CD20, CD22, and CD45 on FL cells and nonneoplastic B-cells.

Notes: MFI of various surface markers was determined and analyzed statistically as described (see Material and methods). The differences between the means, $\pm \mathrm{I}$ standard error of the mean (SEM), for the MFI of each of the antigens (eg, CDI9, CD20, CD22, CD45) is shown. (A value of zero would indicate no difference, or a cell type ratio [lymphoma/ normal] of I, ie, $100=1$ ). 
Table I Immunoglobulin light and heavy chain expression in follicular lymphoma

\begin{tabular}{lllllllll}
\hline Ig (\# cases) & IgM & IgG & IgA & IgD & IgG/IgM & IgM/IgD & ND & Total \\
\hline $\mathrm{K}$ & $\mathrm{I}$ & 6 & $\mathrm{I}$ & 0 & 0 & $\mathrm{I}$ & $\mathrm{I}$ & $23(56 \%)$ \\
$\mathrm{L}$ & 7 & 8 & 0 & 0 & 2 & 0 & 0 & $17(41 \%)$ \\
$\mathrm{K} / \mathrm{L}(-)$ & 0 & $\mathrm{I}$ & 0 & 0 & 0 & 0 & 0 & $\mathrm{I}(2.4 \%)$ \\
Total & $2 \mathrm{I}(52.2 \%)$ & $\mathrm{I}(37.5 \%)$ & $\mathrm{I}(2.5 \%)$ & 0 & $2(5 \%)$ & $\mathrm{I}(2.5 \%)$ & $\mathrm{I}$ & $4 \mathrm{I}$ \\
\hline
\end{tabular}

Abbreviations: ND, heavy chain expression not determined; Ig, immunoglobulin; K, Kappa surface light chain immunoglobulin; L, Lambda surface light chain immunoglobulin; $\mathrm{K} / \mathrm{L}(-)$, Kappa/Lambda surface light chain immunoglobulin negative.

\section{Discussion}

Flow cytometric immunophenotyping is indicated in the diagnosis and classification of non-Hodgkin lymphoma. We report extensive immunophenotypic profiles on 41 nodal FL cases. Our series examines antigens that are not routinely evaluated in cases of $\mathrm{FL}$ and/or have not been thoroughly described in the literature for FL, including CD25, CD103, CD38, $\mathrm{CD} 11 \mathrm{c}$, and detection of immunoglobulin heavy chains. The characteristic immunophenotypic patterns and intensity of antigen expression are useful in identification of FL.

Surface heavy chain immunoglobulin was evaluated routinely in this series. We reported $52 \%$ of cases expressing $\operatorname{IgM}, 38 \%$ expressing $\operatorname{IgG}$, and $3 \%$ expressing $\operatorname{IgA}$; none of the cases expressed IgD only, and this is consistent with previous studies. ${ }^{12,13}$ It has been generally believed that FL cells retain some functional characteristics of normal germinal center cells, such as somatic hypermutation in the variable regions, ${ }^{14-17}$ additionally, immunoglobulin heavy chain class switching reportedly occurs in $\sim 40 \%$ of cases. ${ }^{18}$ Our heavy chain expression data suggest the class switching occurs in approximately half of cases. Interestingly, two of our cases (5\%) showed two subpopulations of FL cells with lambda light chain restriction, but expressing $\operatorname{IgG}$ or IgM heavy chain, respectively. Although rare, cases containing both IgM and IgG expressing FL cells have been described previously. ${ }^{19-21}$ The mechanism for this observation is somewhat controversial. Some authors hypothesized that the co-existing IgM and IgG expressing FL cells indicate that the class-switch mechanism, like the somatic hypermutation mechanism, remains active in FL cells,${ }^{19}$ other studies ${ }^{1,21,22}$ suggested that hypermutation and class switching mechanisms may be active primarily early in the development of lymphoma. ${ }^{23}$ One case in our series showed both IgM and dim IgD expression, and these cells also expressed CD10 and CD38. Co-expression of $\operatorname{IgM}$ and $\operatorname{IgD}$ has been reported previously in $16 \%$ of the FL cases ${ }^{21}$ in which somatic hypermutation was identified. $\operatorname{IgM}(+) / \operatorname{IgD}(+) / C D 38(+)$ cells are considered to represent the earliest germinal center cells that harbor low levels of somatic hypermutation. ${ }^{24}$ Therefore, this unique case in our series may represent FL cells arising at the early germinal center stage.

Of the FL cases in our series, $54 \%$ were $\mathrm{CD} 23$ positive, which is lower than what is reported in the literature..$^{25,26}$ Between $70 \%-78 \%$ of nodal FLs are reported to be CD23 positive ${ }^{25,26}$ however, lower percentages are reported in peripheral blood and bone marrow samples $(43 \%){ }^{27}$ Olteanu et al showed that CD23 expression in FLs was associated with a better overall and event free survival, and CD23(-) FLs were more commonly seen in grade 3 cases. $^{28}$ In our study, 1 of 2 grade 3 cases were CD23(+); due to low numbers of grade 3 cases, we were not able to meaningfully evaluate CD23 expression between the low and high histologic grade cases. We assessed CD23 expression with an antibody combination in which the FLs could not be precisely defined (FMC7/CD79b/CD19/CD23). This would result in a potentially lower sensitivity of detection of CD23 in FL cells, especially if FL cells are not abundant within the sample, and may, in part, explain the lower CD23 positive rate observed in our series.

CD19 expression in FL cells is decreased in intensity when compared to that of reactive germinal center cells; previously, it has been observed that CD19 expression in CD10(+) FL- B-cells is dimmer than that of CD10(-) B-cells within the same sample, ${ }^{8}$ although this finding did not reach statistical significance. In our series, we also demonstrated less intense CD19 expression on CD10(+) FL B-cells than that of CD10(-) non-FL B-cells $(P=0.033$, mean ratio of CD19 MFI between CD10(+) FL and CD10(-) non-FL B-cells is 0.847 ). This finding may be potentially helpful in distinguishing FL from follicular hyperplasia (where CD19 expression of B-cells is of normal intensity), and in instances where lymphoma cells lack surface light chain expression, precluding definitive identification of monoclonal light chain expression.

Brighter CD20 expression in FL cells and reactive follicle center cells has been well documented previously. Recent studies $^{8,14}$ have shown that CD20 expression in FL cells is 
brighter than that of reactive follicle center cells. In our study, we compared the MFI of CD20, CD22, and CD45, between FL and non-FL B-cells. Both CD45 and CD20 expression was consistently stronger in FL cells, even in cases of FLs where CD10 expression was dim or negative; on the other hand, CD22 expression on FL cells showed no significant difference from bystander normal B-cells. The MFI intensity of CD20 expression also showed a positive correlation with the histologic grade 2 versus grade 1 ( $P=0.0023)$. Interestingly, a similar correlation was also seen in the MFI of CD20 of normal B cells and histologic grade $(P=0.019)$. The reason for this observation is not clear, and may be attributed to the microenvironment in LN involved by higher grade FL or due to overlap in analysis gates of FL and non-FL cells. However, it does not diminish the correlative relationship between CD20 intensity of expression in FL cells and histologic grade.

CD10 is a 90 - to 110 -kd monomeric integral membrane glycoprotein expressed in a variety of cell types. It has been well-recognized as a phenotypic feature of follicle center cells, and is often used as gating strategy to define neoplastic cells in FL cases. It has been reported that CD10 expression is decreased in grade 3 and in the interfollicular infiltrate of FL. ${ }^{29}$ Furthermore, CD10 expression is higher on the neoplastic cells of FL than in the (germinal center) B-cells of reactive follicular hyperplasia. ${ }^{30}$ In our series, the CD10 expression was detected in approximately $98 \%$ of the FL cases, and no significant difference in CD10 expression intensity by MFI was detected between histologic grade 1 and grade 2 . These findings are consistent with the previous published studies. ${ }^{29,30}$ Although our series had too few grade 3 cases for meaningful analysis, we did observe that one of the grade $3 \mathrm{FL}$ cases was negative for CD10. Since almost all of the cases from our series had more than $80 \%$ of CD10+ tumor B-cells (suggesting diffuse involvement rather than partial involvement), we were not able to consistently compare the MFI of CD10 between FL cells and cells from normal germinal centers. This may be of interest in further studies, to determine if CD10 MFI could distinguish FL cells from reactive germinal center hyperplasia.

CD38 is a trans-membrane glycoprotein that is widely expressed on hematopoietic cells, and its strong expression is considered another marker for germinal center B-cells. ${ }^{24}$ Recently, decreased CD38 expression on FL cells in comparison to that of reactive germinal center B-cells ${ }^{9}$ has been reported, although no significant difference of CD38 MFI has been observed among the histologic grades. Furthermore, it has been suggested that a new antibody combination of CD44 and CD38 is useful in defining the FL cells, and FL cells usually show weaker CD44 with weaker or equivalent expression of CD38 in comparison to mature, nonneoplastic B cells. ${ }^{31}$ We observed that CD38 was commonly expressed in FL; $98 \%$ of our cases (39 of 40) showed varying degrees of CD38 expression.

Our study was designed to compare FL cells to bystander, nongerminal center B-cells in the same sample, as an internal control. The normal B-cell counterpart of the FL cell is the germinal center cell. Accordingly, the immunophenotypic differences between cells of nodal FL and germinal center cells of reactive nodal hyperplasia have been previously studied and are thoroughly described. ${ }^{8,9}$ However, from a practical standpoint, a clinical specimen received for FC testing may often contain an overwhelmingly large proportion of FL cells, and normal germinal center cells may not be detectable, or if present, may be low in number and difficult to detect reliably as an internal standard. Our series showed that an average of $88 \%$ of the B-cells in our samples were FL; however, detection of bystander nongerminal center B-cells were readily apparent by FC, even though they comprised the minority of B-cells in the sample, and served as a useful internal control within the sample. Virtually all LN samples contain residual nonneoplastic B-cells, making this population an ideal internal standard for the clinical laboratory, as per standard practice, as variations exist from patient to patient and sample to sample. Using this internal standard, we demonstrate that FL cells express brighter CD20, brighter CD45 and dimmer CD19 than nonneoplastic B-cells. Our findings appear to differ from the previously described literature, ${ }^{8,9}$ but are actually complementary to them, because the design of our study was different.

In summary, we provide extensive immunophenotypic profiles in cases of nodal FL by FC, including nonconventional antigens and distribution of heavy chain immunoglobulin expression. We show that increased CD20, CD45 are characteristic features of nodal FL, when compared to bystander nongerminal center B-cells as an internal control, and confirm previous reports that diminished CD19 expression is a distinguishing characteristic of FL. We observed unusual heavy chain coexpression patterns, including IgM and IgG, or IgM and IgD expression on FL cells in select cases. Furthermore, increased CD20 expression on FL cells was associated with histologic grade 2 versus 1 .

FC assessment of MFI can detect subtle changes in antigen expression that may not be detected by visual 
examination of FC dot plots or visual examination of immunohistochemistry. Using MFI, we detected subtle immunophenotypic differences in nodal FL that were not apparent by simple visual inspection of FC scatter plots. Using MFI may be a means of uncovering subtle but unique immunophenotypic features in well-known diseases such as FL, as well as less newly emerging neoplastic hematolymphoid entities.

\section{Acknowledgments}

This research was supported by the Intramural Research Program of the NIH, NCI.

\section{Disclosure}

The authors have no conflicts of interest to report.

\section{References}

1. Swerdlow SH, Campo E, Harris NL, et al. WHO Classification of Tumours of Haematopoietic and Lymphoid Tissues, Fourth Edition. Geneva, Switzerland: World Health Organization; 2008.

2. Ott G, Katzenberger T, Lohr A, et al. Cytomorphologic, immunohistochemical, and cytogenetic profiles of follicular lymphoma: 2 types of follicular lymphoma grade 3. Blood. 2002;99(10):3806-3812.

3. Ott G, Rosenwald A. Molecular pathogenesis of follicular lymphoma. Haematologica. 2008;93(12):1773-1776.

4. Roulland S, Navarro JM, Grenot P, et al. Follicular lymphoma-like B cells in healthy individuals: a novel intermediate step in early lymphomagenesis. J Exp Med. 2006;203(11):2425-2431.

5. Kussick SJ, Kalnoski M, Braziel RM, Wood BL. Prominent clonal B-cell populations identified by flow cytometry in histologically reactive lymphoid proliferations. Am J Clin Pathol. 2004;121(4):464-472.

6. Arun I, Wulu JA, Janik JE, et al. Visual inspection versus quantitative flow cytometry to detect aberrant $\mathrm{CD} 2$ expression in malignant $\mathrm{T}$ cells. Cytometry B Clin Cytom. 2010;78(3):169-175.

7. Jasper GA, Arun I, Venzon D, et al. Variables affecting the quantitation of CD22 in neoplastic B cells. Cytometry B Clin Cytom. 2011;80(2):83-90.

8. Ray S, Craig FE, Swerdlow SH. Abnormal patterns of antigenic expression in follicular lymphoma: a flow cytometric study. Am J Clin Pathol. 2005;124(4):576-583.

9. Mantei K, Wood BL. Flow cytometric evaluation of CD38 expression assists in distinguishing follicular hyperplasia from follicular lymphoma. Cytometry B Clin Cytom. 2009;76(5):315-320.

10. Jiang L, Yuan CM, Hubacheck J, et al. Variable CD52 expression in mature T cell and NK cell malignancies: implications for alemtuzumab therapy. Br J Haematol. 2009;145(2):173-179.

11. Borowitz MJ, Bray R, Gascoyne R, et al. US-Canadian Consensus recommendations on the immunophenotypic analysis of hematologic neoplasia by flow cytometry: data analysis and interpretation. Cytometry. 1997;30(5):236-244.

12. Harris NL, Nadler LM, Bhan AK. Immunohistologic characterization of two malignant lymphomas of germinal center type (centroblastic/ centrocytic and centrocytic) with monoclonal antibodies. Follicular and diffuse lymphomas of small-cleaved-cell type are related but distinct entities. Am J Pathol. 1984;117(2):262-272.
13. Jaffe ES, Harris NL, Vardiman JW, Campo E, Arber D. Hematopathology, 1st ed. Philadelphia, PA: Elsevier; 2011.

14. Ottensmeier CH, Thompsett AR, Zhu D, Wilkins BS, Sweetenham JW, Stevenson FK. Analysis of VH genes in follicular and diffuse lymphoma shows ongoing somatic mutation and multiple isotype transcripts in early disease with changes during disease progression. Blood. 1998;91(11):4292-4299.

15. Cleary ML, Meeker TC, Levy S, et al. Clustering of extensive somatic mutations in the variable region of an immunoglobulin heavy chain gene from a human B cell lymphoma. Cell. 1986;44(1):97-106.

16. Zelenetz AD, Chen TT, Levy R. Clonal expansion in follicular lymphoma occurs subsequent to antigenic selection. JExp Med. 1992;176(4): 1137-1148.

17. Bahler DW, Levy R. Clonal evolution of a follicular lymphoma: evidence for antigen selection. Proc Natl Acad Sci U SA. 1992;89(15): 6770-6774.

18. Vaandrager JW, Schuuring E, Kluin-Nelemans HC, Dyer MJ, Raap AK, Kluin PM. DNA fiber fluorescence in situ hybridization analysis of immunoglobulin class switching in B-cell neoplasia: aberrant $\mathrm{CH}$ gene rearrangements in follicle center-cell lymphoma. Blood. 1998;92(8):2871-2878.

19. Zelenetz AD, Chen TT, Levy R. Histologic transformation of follicular lymphoma to diffuse lymphoma represents tumor progression by a single malignant B cell. J Exp Med. 1991;173(1):197-207.

20. Raghoebier S, Broos L, Kramer MH, et al. Histological conversion of follicular lymphoma with structural alterations of $\mathrm{t}(14 ; 18)$ and immunoglobin genes. Leukemia. 1995;9(10):1748-1755.

21. Aarts WM, Bende RJ, Steenbergen EJ, et al. Variable heavy chain gene analysis of follicular lymphomas: correlation between heavy chain isotype expression and somatic mutation load. Blood. 2000;95(9): 2922-2929.

22. Aarts WM, Bende RJ, Bossenbroek JG, Pals ST, van Noesel CJ. Variable heavy-chain gene analysis of follicular lymphomas: subclone selection rather than clonal evolution over time. Blood. 2001;98(1):238-240.

23. Jaffe ES, Harris NL, Vardiman JW, Camp E, Arber D. In: Schmitt W, Vosburgh A, editors. Hematopathology, 1st ed. Philadelphia, PA: Elsevier; 2011

24. Liu YJ, Arpin C. Germinal center development. Immunol Rev. 1997; 156(1):111-126.

25. Garcia DP, Rooney MT, Ahmad E, Davis BH. Diagnostic usefulness of CD23 and FMC-7 antigen expression patterns in B-cell lymphoma classification. Am J Clin Pathol. 2001;115(2):258-265.

26. XuY, McKenna RW, Asplund SL, Kroft SH. Comparison of immunophenotypes of small B-cell neoplasms in primary lymph node and concurrent blood or marrow samples. Am J Clin Pathol. 2002;118(5):758-764.

27. Gujral S, Polampalli SN, Badrinath Y, et al. Immunophenotyping of mature B-cell non Hodgkin lymphoma involving bone marrow and peripheral blood: critical analysis and insights gained at a tertiary care cancer hospital. Leuk Lymphoma. 2009;50(8):1290-1300.

28. Olteanu H, Fenske TS, Harrington AM, Szabo A, He P, Kroft SH. CD23 expression in follicular lymphoma: clinicopathologic correlations. Am J Clin Pathol. 2011;135(1):46-53.

29. Eshoa C, Perkins S, Kampalath B, Shidham V, Juckett M, Chang CC. Decreased CD10 expression in grade III and in interfollicular infiltrates of follicular lymphomas. Am J Clin Pathol. 2001;115(6):862-867.

30. Almasri NM, Iturraspe JA, Braylan RC. CD10 expression in follicular lymphoma and large cell lymphoma is different from that of reactive lymph node follicles. Arch Pathol Lab Med. 1998;122(6):539-544.

31. Detry G, Drenou B, Ferrant A, et al. Tracking the follicular lymphoma cells in flow cytometry: characterisation of a new useful antibody combination. Eur J Haematol. 2004;73(5):325-331. 


\section{Publish your work in this journal}

Pathology and Laboratory Medicine International is a peer-reviewed, open access journal focusing on innovative basic research and translational research related to pathology or human disease. The journal includes original research, updates, case reports, reviews and commentaries on current controversies. The Academic Sponsor

of this journal is the Chinese American Pathology Association (CAPA). The manuscript management system is completely online and includes a very quick and fair peer-review system. Visit http://www.dovepress.com/testimonials.php to read real quotes from published authors.

Submit your manuscript here: http://www.dovepress.com/pathology-and-laboratory-medicine-international-journa 